\title{
The studying of hybrid line with spherical grains and reduced height obtained by crossing triticale and synthetic hexaploid wheat
}

\author{
Zorina M.V. ${ }^{1,2 *}$, Adonina I.G. ${ }^{2}$, Mehdiyeva S.P. ${ }^{3}$, Salina E.A. ${ }^{4}$ \\ ${ }^{1}$ Novosibirsk State University, Novosibirsk, Russia \\ ${ }^{2}$ Institute of Cytology and Genetics, SB RAS, Novosibirsk, Russia \\ ${ }^{3}$ Genetic Resources Institute of ANAS, Azerbaijan \\ ${ }^{4}$ Kurchatov Genomic Center of the Institute of Cytology and Genetics, SB RAS, Novosibirsk, Russia \\ * email: mariamnazorina@gmail.com
}

Spherical grains and reduced height was identified in a hybrid line 1102. These features are valuable in agriculture. The line 1102 was obtained in Genetic Resources Institute of ANAS by crossing triticale ABR $(2 n=6 x=42)$ and synthetic hexaploid wheat ADS $(2 n=6 x=42)$. The karyotype of this line was studied using the GISH and FISH methods; it corresponds to the karyotype of hexaploid wheat $(2 n=6 x=42$, BBAADD). Whole rye chromosomes and translocations were not found in the studied line. Spelt 1 - repeat blocks are found at the ends of the long arms of chromosomes 3B. It may indicate a translocation from Ae. speltoides and requires an additional research. Phenotyping and karyotyping of individual plants of line 1102 showed its homogeneity. The analysis of cultivars of common wheat by grain shape and stem length was carried out in order to select samples for crossing with short-stemmed, round-grain line 1102. The grain shape was assessed by the SeedCounter program. Line 1102 was crossed with spring varieties of common wheat, Kinelskaya 40 and Lutescens 85, which are the most contrasting with it in terms of the studied features to obtain a mapping population for determine the genetic basis of reduced height and spherical grains in the studied line. Moleculargenetic analysis of line 1102 was conducted using markers specific to the already known dwarfing genes (Rht-B1, Rht-D1, Rht-8, Rht-9).

Acknowledgments: This work was carried out as a part of the budget project No. 02592021-0012 and was funded by RFBR, project No. 20-016-00122. 